
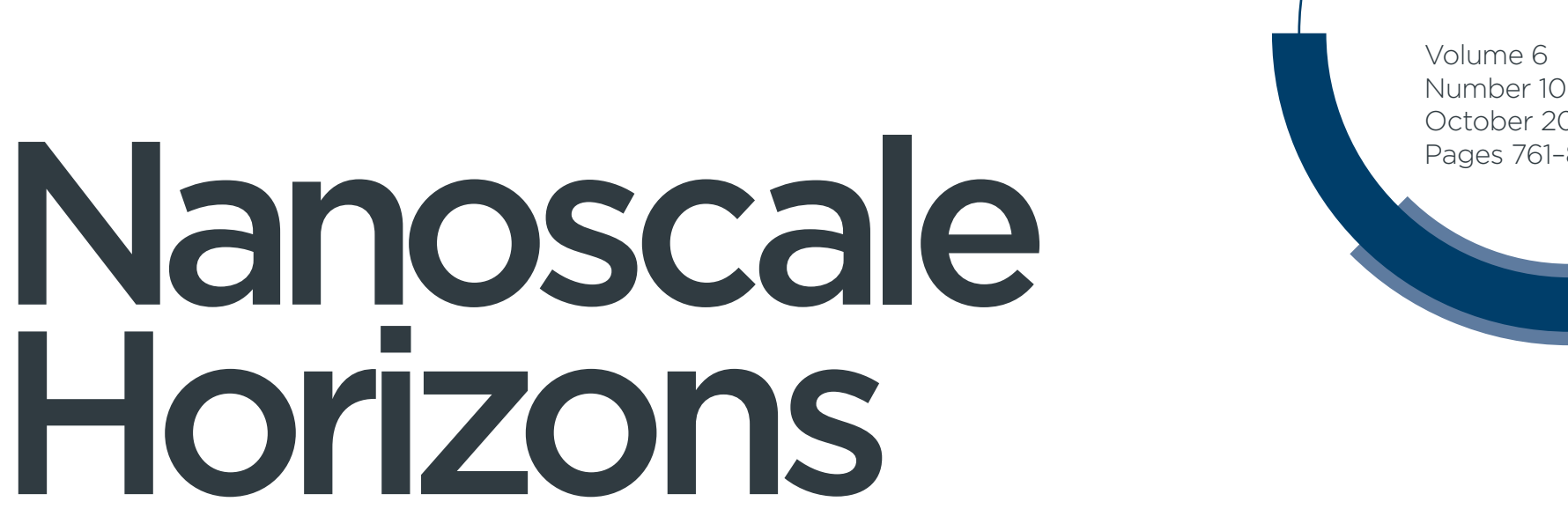

Number 10

October 2021

Pages 761-832

The home for rapid reports of exceptional significance in nanoscience and nanotechnology rsc.li/nanoscale-horizons

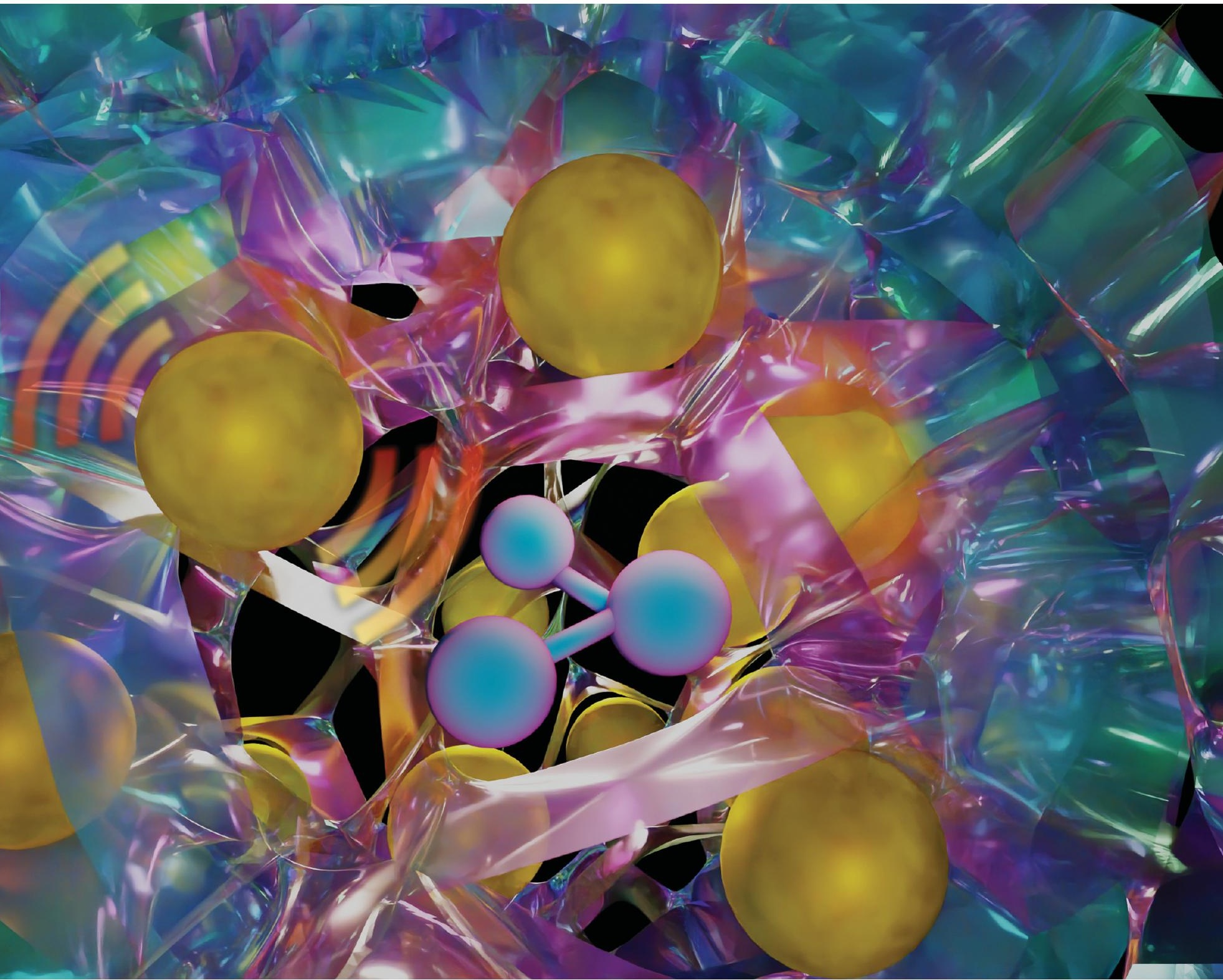


Check for updates

Cite this: Nanoscale Horiz., 2021, 6,781

Received 21st April 2021

Accepted 14th July 2021

DOI: $10.1039 / \mathrm{d} 1 \mathrm{nh} 00228 \mathrm{~g}$

rsc.li/nanoscale-horizons

\section{Gold nanoplasmonic particles in tunable porous silicon 3D scaffolds for ultra-low concentration detection by SERS $\uparrow$}

\author{
Rihan Wu, $\ddagger^{\mathrm{a}}$ Qihao Jin, $\ddagger_{\ddagger^{\mathrm{b}}}$ Catherine Storey, ${ }^{\mathrm{a}}$ Jack Collins, ${ }^{a}$ \\ Guillaume Gomard, (D) ${ }^{c}$ Uli Lemmer, ${ }^{b}$ Leigh Canham, ${ }^{a}$ Rainer Kling ${ }^{b}$ and \\ Andrey Kaplan (D) *a
}

\begin{abstract}
A composite material of plasmonic nanoparticles embedded in a scaffold of nano-porous silicon offers unmatched capabilities for use as a SERS substrate. The marriage of these components presents an exclusive combination of tightly focused amplification of Localised Surface Plasmon (LSP) fields inside the material with an extremely high surface-to-volume ratio. This provides favourable conditions for a single molecule or extremely low concentration detection by SERS. In this work the advantage of the composite is demonstrated by SERS detection of Methylene Blue at a concentration as low as a few picomolars. We systematically investigate the plasmonic properties of the material by imaging its morphology, establishing its composition and the effect on the LSP resonance optical spectra.
\end{abstract}

Localised Surface Plasmon (LSP) is a coherent oscillation of electrons at the interface between metallic nanoparticles and the surrounding medium. LSP can be excited by light when its frequency matches that of the oscillations, producing a strong enhancement of the electromagnetic field in the vicinity of the particles under the conditions of the resonance. ${ }^{1}$ The LSP Resonance (LSPR) is distinguished by intense absorption and scattering of light. Its wavelength and intensity are primarily determined by the type of material, size, shape, environment refractive index and coupling between adjacent nanoparticles. ${ }^{2-4}$ In the past few decades, extensive studies have been conducted to adopt LSPR in biological and chemical sensing systems due to its high sensitivity to the surrounding environment., The ability of photon-trapping and strong absorption of LSPR

\footnotetext{
${ }^{a}$ School of Physics and Astronomy, University of Birmingham, Birmingham B15 2TT, UK. E-mail: a.kaplan.1@bham.ac.uk

${ }^{b}$ Light Technology Institute, Karlsruhe Institute of Technology, Engesserstrasse 13, 76131 Karlsruhe, Germany

${ }^{c}$ Carl Zeiss AG, ZEISS Innovation Hub@KIT, Hermann-von-Helmholtz-Platz 6,

76344 Eggenstein-Leopoldshafen, Germany

$\dagger$ Electronic supplementary information (ESI) available. See DOI: 10.1039/ d1nh00228g

\# Contributed equally to this work.
}

\begin{abstract}
New concepts
We developed a new substrate for on-chip Surface Enhanced Raman Spectroscopy. The substrate surpasses known alternatives and provides signal enhancement by a factor of $10^{9}$, achieving a single-molecule detection level. This property has been corroborated by detecting Raman scattering from an analyte with picomolar $\left(10^{-12} \mathrm{M}\right)$ concentration of methylene blue dye. To achieve the break-through quality, we developed a new method to embed gold nanoclusters into a 3D scaffolding of nano-porous silicon (npSi). This new composite material combines unique properties of both components. The role of gold nanoclusters is to enhance the light field by means of Localised Surface Plasmon (LSP) resonance excitation. The function of the $\mathrm{npSi}$ dielectric environment is to tighten further the localisation and provide an enormous area exposed to LSP. Thus, analyte molecules adsorbed on the pores have an exceedingly high chance to interact with the tightlyfocused LSP and participate in enhanced Raman scattering. The material applications spread far beyond SERS because it combines the rich world of nanoplasmonics with the high-tech of semiconductors. Plasmonics materials have excellent absorption cross-section, high electron concentration and tunability, the properties sought to enhance the performance of semiconductor detectors, lasers and imaging sensors. We are hopeful that our development will advance significantly the implementation of plasmonic/semiconductor composites into industrial applications.
\end{abstract}

at the resonance wavelength have further promoted its implementation in light harvesting devices. ${ }^{7,8}$ Other fields of research and applications extend to metamaterials, ${ }^{9}$ plasmonic devices, ${ }^{10,11}$ diagnosis and treatment of diseases. ${ }^{12-14}$

Several types of LSPR substrates with nanoparticles in colloidal suspension, deposited on the surface and embedded in a dielectric matrix have been developed and investigated. ${ }^{15-17}$ The former two types are most commonly used in biological and chemical sensing systems, implementing Surface Enhanced Raman Spectroscopy (SERS), where the analytes are either mixed with colloidal solution or in contact with the substrate surface. ${ }^{18}$ The LSPR induced by nanoparticles localises and enhances the electromagnetic field which amplifies the Raman signal of the surrounding molecules. ${ }^{19}$ In the latter type, metallic 
nanoparticles are usually embedded into a nonconducting solid state environment, such as silicon oxide or organic polymers. ${ }^{20}$ These hybrid composite materials not only preserve the LSPR response of the metal nanoparticles from degradation, but also improve the robustness and responsiveness, thereby further expanding their application range. The use of plasmonic nanoparticles was extended to Au@Pt core-shell nanostructures facilitating dual functionalities to track Pt-catalysed reactions and quantitatively analyse the response by SERS. The particles preserve their morphological uniformity as revealed by TEM imaging after a few cycles of recycling following the particles' use in a catalytic reaction. ${ }^{21}$ Although there are many advantages of hybrid bulk composites over solution/surface counterparts, technological limitations to embed metallic nanoparticles into the matrix of bulk materials, in particular semiconductors, remain unresolved, because of difficulties involved in the fabrication process. The conventional mixing methods, such as melt quenching, ${ }^{22}$ colloidal method ${ }^{23}$ and metal-dielectric co-sputtering, ${ }^{24}$ are either not suitable or have not been developed to an adequate level suitable for the large-scale and low-cost fabrication of nanoplasmonic-semiconductor composites. Nevertheless, recent studies have revealed fascinating properties and perspectives of nanoplasmonic-semiconductor composites for enhanced efficiency of photocatalysis, ${ }^{25,26}$ as well as demonstration of a mechanism of plasmon-induced hot carrier generation and their migration through a Schottky barrier of $\mathrm{Au} / \mathrm{TiO}$ dumbbell structures, enhancing the photocatalytic performance. $^{27}$ Other examples of the composites' applications include amplifications of photoluminescence, ${ }^{28}$ solar energy conversion $^{29}$ and optical nonlinearities. ${ }^{30}$

In this work, we aim to develop a method scalable for the production of large quantities of nanoplasmonic-semiconductor composites exhibiting high uniformity. Nano-porous silicon (np-Si) was selected as the semiconductor matrix due to its low manufacturing cost, ${ }^{31}$ robustly developed fabrication process and in-depth documented optical and electronic properties. $^{32,33}$ Moreover, np-Si provides a natural template for metallic nanoparticle growth inside the interconnected nanosized pore channels. We developed a method of spontaneous growth of $\mathrm{Au}$ nanoparticles (AuNPs) inside np-Si using the immersion plating technique. This process demands precise control of the solution temperature and concentration to ensure uniform growth of $\mathrm{Au}$ particles inside the pores, while concurrently minimising unwanted particle clustering on the $\mathrm{np}$-Si facets. We fabricated samples with different volume fractions of Au nanoparticles and examined their composition, morphology and uniformity by means of Scanning Electron Microscopy (SEM) and Transmission Electron Microscopy (TEM). We show that the nanoparticle size and density distribution can be controlled by varying the deposition time and solution concentration. The presence of the LSPR was observed by dark-field microscope and a VIS-NIR scattering spectrometer. Au/np-Si composites exhibit the LSPR at the red end of the visible spectrum, extending to the near infrared region for the samples with increasing $\mathrm{Au}$ nanoparticle volume fractions.

The sample with the highest Au volume fraction was used as a SERS substrate to measure the Raman spectrum of the
Methylene Blue (MB) benchmark compound. We demonstrate an enhancement factor of $\sim 10^{9}$ under the excitation wavelength of $808 \mathrm{~nm}$ in the vicinity of the LSPR. Such enhancement is suitable for a scenario where a single molecule detection is desired. ${ }^{34,35}$

\section{Results and discussion}

\section{Sample preparation and characterisation}

We adopted a wet chemical synthesis method based on the previously reported studies ${ }^{36,37}$ exploring gold ion reduction from a solution of tetrachloroauric acid $\left(\mathrm{HAuCl}_{4}\right)$ with borohydride as a reducing agent. However, in this work, we adopted a procedure where no reducing agent was required. Instead, hydrogen atoms chemisorbed at the pore surface of np-Si act as a reducing agent during AuNP impregnation (see Methods). Fig. 1(a) wrap-ups the main steps of the sample preparation. A $550 \mathrm{~nm}$-thick np-Si layer with underlaying silicon substrate was pre-treated with $2 \% \mathrm{HF}$ solution for $30 \mathrm{~s}$ to remove the native oxide from the pore surface and to saturate dangling bonds with hydrogen. After the treatment, the samples were immediately immersed in $\mathrm{HAuCl}_{4}$-ethanol solution, where the gold ion reduction occurs at the pore channel surfaces accompanied by aggregation and spontaneous growth of AuNPs. To facilitate the penetration of the solution into the pores, an ultrasonic bath was used during immersion, which not only enhances the inflow, but also prevents the growth and clustering on the top surface. In addition, the solution concentration and environment temperature play important roles in achieving uniform $\mathrm{Au}$ deposition along the depth coordinate. Due to a slow diffusion rate of the solution in np-Si $\left(\sim 10^{-9}-10^{-10} \mathrm{~m}^{2} \mathrm{~s}^{-1}\right),{ }^{31}$ AuNPs tend to grow in the direction from the top to the bottom of the substrate. Therefore, controlling the reduction rate through concentration and temperature is essential to ensure the solution reaches the bottom before AuNP grow at the top, blocking the opening of the pore channel. Through iterative experimentation, it was found that at room temperature, the diffusion and reduction rates are balanced for $\mathrm{HAuCl}_{4}$ concentration not greater than $1 \mathrm{mM}$. At above room temperature the clusters tend to grow on the surface restricting penetration of the solution into the pores and inhibiting uniform growth through the layer thickness. Consequently, we attempted to lower the temperature and increase the flow rate. However, this did not produce reproducible results as it was difficult to maintain the parameters steady without further elaborate development of the set-up. Therefore, we focused on finding a range of concentrations suitable for the impregnation at room temperature. Samples with different densities and sizes of AuNPs were obtained by varying the $\mathrm{HAuCl}_{4}$ concentration and retention time of np-Si in the solution. Table 1 shows the details of the concentration and immersion time of the four samples that were further investigated in this work. The quoted AuNP volume fractions were estimated by modelling the results obtained from angle resolved reflectance measurements (see the ESI $\dagger$ ). After the immersion plating, the samples were rinsed in pure ethanol and gently air dried on a hotplate at $60{ }^{\circ} \mathrm{C}$. 


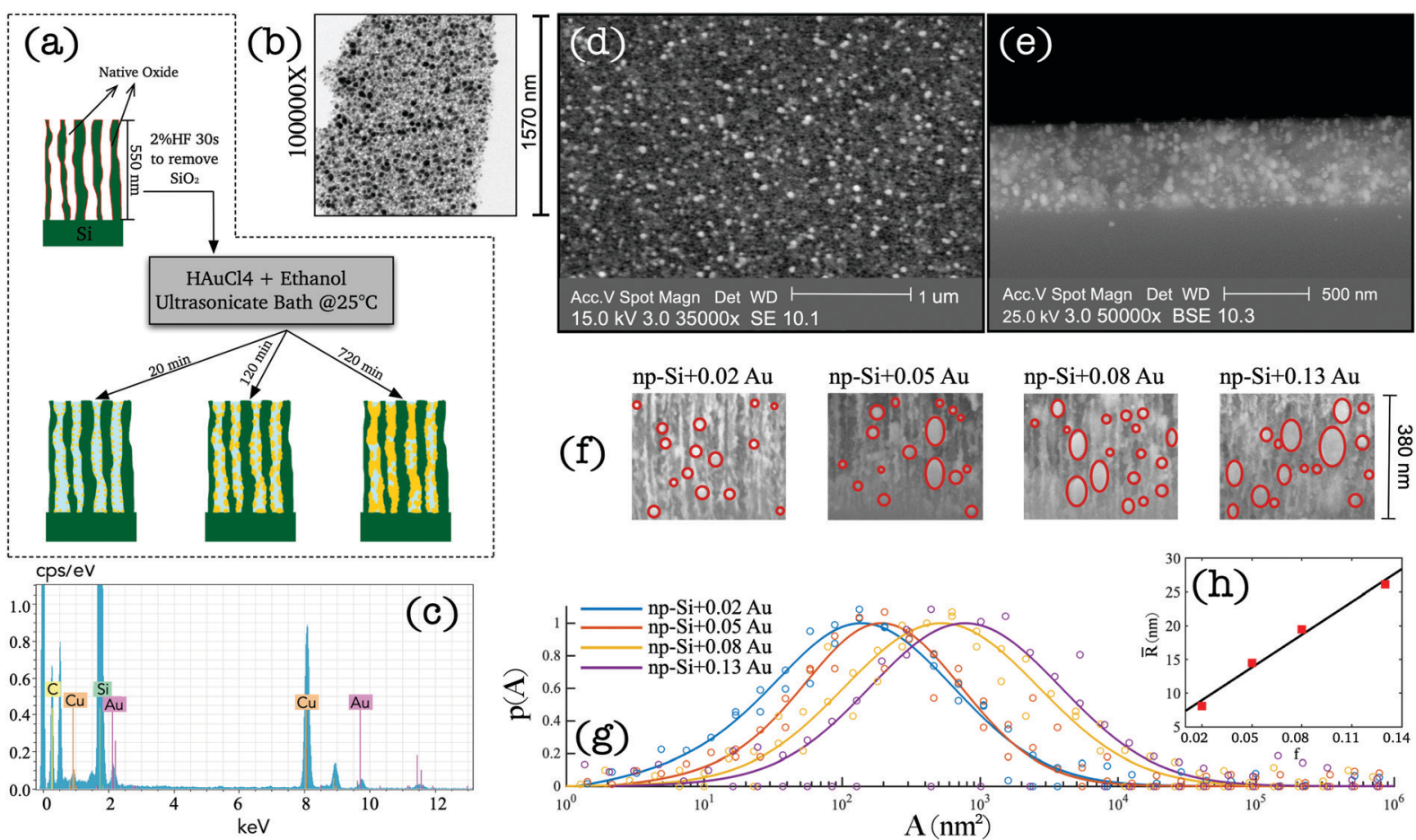

Fig. 1 (a) Schematical presentation of the fabrication procedure of the Au/np-Si composite material; (b) TEM image of a fragment of Au/np-Si; the dark spots are AuNPs and the bright area is np-Si; (c) EDX spectrum of the Au/np-Si composite; (d) top and (e) cross-section views of the SEM image of the $\mathrm{Au} / \mathrm{np}$-Si composite with Au volume fraction of 0.02; (f) zoomed-in cross-section of SEM images for samples with different Au volume fraction, $f$; (g) scaled probability distribution of cross-sectional area $A$; (h) ellipsoidal quadratic mean radius, $\bar{R}$, as a function of AuNP volume fraction, $f$.

Table 1 Parameters and sample information of a gold ion reduction process: $\mathrm{HAuCl}_{4}$ solution concentration, $c$; immersion time, $t$; and $\mathrm{Au}$ volume fraction, $f$. All samples were prepared at the constant temperature of $25^{\circ} \mathrm{C}$

\begin{tabular}{llcl}
\hline Sample & $c(\mathrm{mM})$ & $t(\min )$ & $f$ \\
\hline Au/np-Si 1 & 0.5 & 10 & 0.02 \\
Au/np-Si 2 & 1 & 20 & 0.05 \\
Au/np-Si 3 & 1 & 120 & 0.08 \\
Au/np-Si 4 & 1 & 720 & 0.13
\end{tabular}

A layer of processed np-Si was gently fractured into small fragments to examine under TEM and Energy-Dispersive X-ray (EDX) to verify the presence of AuNPs. Fig. 1(b) shows the TEM image of an arbitrary segment of the composite. The dark spots are the AuNPs surrounded by the bright sponge of the np-Si matrix. It can be seen that some of the pores are fully occupied by $\mathrm{Au}$, while others remain hollow. Fig. 1(c) shows the EDX spectrum of np-Si after the Au impregnation. The pronounced $\mathrm{Au}$ and silicon peaks are clearly observed at their characteristic energies of $2.12,9.71$ and $1.74 \mathrm{eV}$, respectively. ${ }^{38,39}$ We note that the carbon and copper peaks are contributed by the supporting TEM grid.

To demonstrate the uniformity of the cluster distribution along the sample depth, we show the top-view and cross-section SEM images of the Au/np-Si composite with a Au volume fraction of 0.02 in Fig. $1(\mathrm{~d}$ and e), respectively. These results confirm that there is no aggregation of particles on the top surface, while they are evenly spread along the depth of the $550 \mathrm{~nm}$-thick np-Si matrix. Fig. 1(f) shows the zoomed-in view of the SEM cross-sections for four samples with different AuNP volume fractions, $f$. It can be seen that the size of the AuNPs increases with the immersion time, while at the longer immersion times the larger clusters tend to grow anisotropically along channels and form rather elliptical shapes. This preferential direction of the growth is imposed by the pore channels walls. By analysing a large number of SEM cross-section images, we obtained the AuNP cluster cross-sectional area distribution as shown in Fig. $1(\mathrm{~g})$. In addition, the ellipsoidal quadratic mean radius, $\bar{R}$, as a function of Au volume fraction, $f$, is shown in Fig. 1(h). These figures indicate a general correlation for the clusters to grow larger as their density increases.

\section{Localised surface plasmon resonance}

To gain further insight into the nature of Au/np-Si composites, we assessed their optical properties. The samples were imaged by using a real-colour CCD camera through a Dark Field Microscope (DFM) collecting the scattered light. The images are shown in Fig. 2(a-d) where the apparent colour of the scattered light is red. This is in contrast to the typical green colour for similar size AuNPs in air. ${ }^{40}$ This redshift is attributed to the renormalisation of the LSPR wavelength according to the Fröhlich resonance condition ${ }^{41}$ for which the Drude dielectric function of gold nanoparticles, $\varepsilon_{\text {AuNP }}$, matches that of the environment according to $\varepsilon_{\mathrm{AuNP}}=-2 \varepsilon_{\mathrm{env}}$. For particles in $\mathrm{np}$-Si, the surrounding effective dielectric function, $\varepsilon_{\mathrm{env}}$, is 

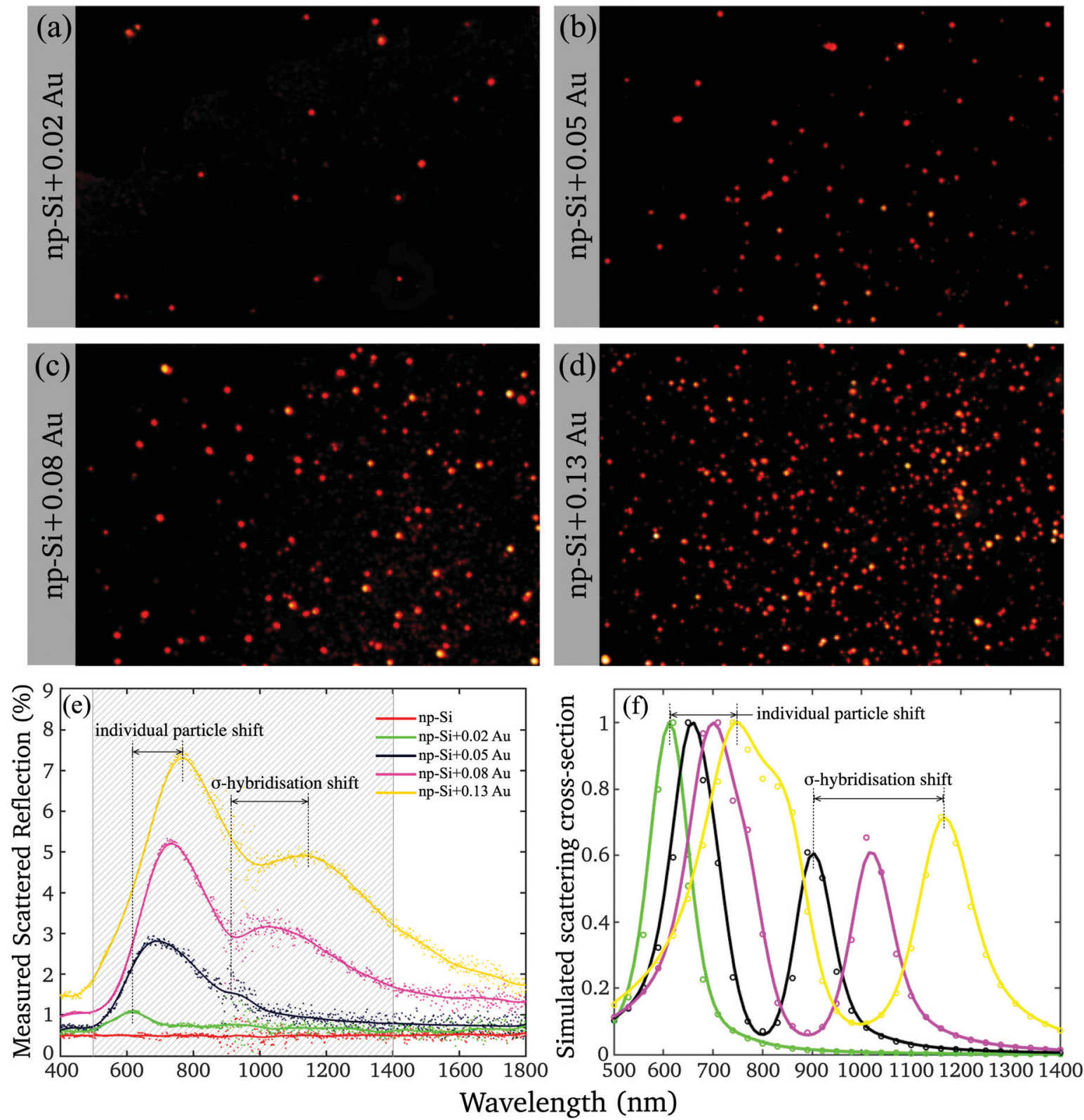

Fig. 2 (a-d) Dark Field Microscope (DFM) images of the four samples with different Au volume fractions; (e) measured scattered reflection of np-Si samples without and with different volume fraction of $\mathrm{Au}$; (f) simulated scattering cross-section (normalised) for the fitting parameters listed in Table 2.

nearly twice larger than that of air, resulting in the resonance conditions rearranged towards longer wavelengths.

Yet, the DFM observation of the scattering has a few limitations. The CCD camera imposes the cut-off wavelength of $650 \mathrm{~nm}$ and integrates the spectrum while missing the image of the particles scattering at the longer wavelength. The DFM imaging also tends to grossly overrepresent the high-end tail of the physical cross-section distribution (Fig. 1(g)), while uncounting for the clusters with the dimensions smaller than the microscope resolution limit.

The deficiency was resolved by means of the wavelengthresolved light scattering measurement over an extended VIS-NIR spectral range (see Methods). Fig. 2(e) shows the scattering spectra of np-Si without and with different volume fractions of Au. The scattering spectrum of the np-Si sample without AuNPs is almost flat as its roughness parameter, governed by the pore dimensions, is much smaller than the wavelengths of the spectrum and there are no available scattering centres able to deflect the incoming photons from the original path. However, adding just a minute Au volume fraction of 0.02 to the np-Si results in the appearance of the scattering band peaked around $600 \mathrm{~nm}$, attributed to the LSPR. As the Au volume fraction increases, the resonance peak further shifts to the NIR region, accompanied by the development of the second scattering band, covering the wavelength region between 1000 and $1300 \mathrm{~nm}$. There are two main physical effects that are likely involved in the alteration of the spectrum by increasing the Au fraction in the material. 
First, the plasmon resonance shifts towards longer wavelength as the AuNP size increases. ${ }^{42}$ This is very likely attributed to the change in the local environment surrounding individual particles. While a particle grows, it fills the available space expanding simultaneously towards the opposite wall and along the channel. Hence, the size of a particle determines the fractions of silicon and air in its surrounding. The smaller and round particles are more exposed to air and less to silicon at the interface of the contact area between the pore wall and gold. For larger particles the contact area with silicon increases because of their elliptical shape filling the channel. Hence, the larger particles are locally surrounded by the environment with a higher effective dielectric function and their LSPR wavelength shifts to red with respect to the smaller ones.

Second, the plasmon coupling between adjacent AuNPs can cause the split of the plasmon mode. ${ }^{43,44}$ Using the AuNPs density and mean radius, it can be estimated that for the lowest fraction, the mean spacing between adjacent AuNPs is relatively large, $>10 \mathrm{~nm}$ (Fig. S3, ESI $\dagger$ ). Thus, for smaller particles and lower densities the interaction is diminishing, because the plasmon field decays over the factor of $1 / e$ within the distance of $<5 \mathrm{~nm} .{ }^{45}$ Under these conditions the AuNPs can be treated as isolated clusters surrounded by an np-Si environment. However, at the greater Au volume fractions, which yield larger particles, the spacing between particles is smaller than the field extension and the coupling effect can no longer be ignored. At the larger AuNP densities, the coupling produces an additional plasmon resonance corresponding to the $\sigma$-hybridisation of two individual AuNP particles with collinear and unidirectional fields. ${ }^{43}$ This mode energy is lower than that of a single particle, because of the attractive nature of plasmon-plasmon interaction and it appears in the spectrum at longer wavelength than that of an individual particle. Since the AuNPs in the matrix have rather distributed sizes and spacings, their scattering spectra are composed of weighted contributions from single and hybridised plasmon modes.

We note here that, although the spectra demonstrate a red shift as Au volume fraction increases, the clusters colour remains almost unchanged in the DFM images (Fig. 2(a-d)), because the cut-off wavelength and integration smear the effect.

To gain a quantitative understanding of the observed LSPR spectra, we used the finite element method to simulate the scattering spectra of AuNPs embedded in an np-Si environment (see Methods). The simulation takes as an input of the mean radius, $\bar{R}$, and spacing, $\bar{d}$, between gold particles surrounded by np-Si. $\bar{R}$ and $\bar{d}$ were determined from the SEM images, as outlined above. The dielectric function of gold was used from the literature. ${ }^{46}$ The effective dielectric function of the np-Si environment (without $\mathrm{Au}$ inclusions), $\varepsilon_{\mathrm{env}}$, was estimated by the use of the Bruggeman model: ${ }^{47,48}(1-p) \frac{\varepsilon_{\text {air }}-\varepsilon_{\text {env }}}{\varepsilon_{\text {air }}+2 \varepsilon_{\text {env }}}+$ $p \frac{\varepsilon_{\mathrm{si}}-\varepsilon_{\mathrm{env}}}{\varepsilon_{\mathrm{si}}+2 \varepsilon_{\mathrm{env}}}=0$, where $p$ is the volume fraction of silicon and $\varepsilon_{\text {air }}=1 . p$ is an adjustable parameter, which accounts for the fact that $\varepsilon_{\mathrm{env}}$ depends on the size of gold particles in the pore channel, as discussed previously.
Three main assumptions were made for the simulation: (1) AuNPs are surrounded by a homogeneous environment with the dielectric function of $\varepsilon_{\mathrm{env}}$, according to the effective media approximation with adjustable parameter, $p$; (2) the refractive index of AuNP is the same as that of bulk Au; (3) AuNPs have a spherical shape. Although, the actual shape of the particles is that of a spheroid, they are predominantly oriented with their major axis along the channels and depth coordinate. Hence, at the normal light incidence, light is polarised in the plane of the circular cross-section containing the minor axis.

The simulation results of the normalised scattering crosssection are shown in Fig. 2(f) and the corresponding parameters of the calculations are listed in Table 2 . It can be seen that the modelling essentially reproduces the spectral locations of the single particle and $\sigma$-hybridisation resonances, as well as the tendency of the former to dominate. The simulation does not include sources of the possible resonance broadening, such as AuNP size and space distribution, deviation from the perfect spherical shape, dependence of the resonance life time on the particle size and contribution from the multipole interactions. Yet, it supports our assumption that the shift of the higher energy resonance (single particle) is proportional to the dielectric function of the effective np-Si environment. While the lower energy resonance depends on the strength of the coupling of an attractive dipole-dipole interaction as $d^{-3}$.

The scattering cross-section, $C_{\text {sca }}$, can be estimated from the measurements of the fractional scattering intensity and compared to the simulation. $I_{\text {sca, the following: }}{ }^{49} C_{\text {sca }}=$ $-\ln \left(I_{\text {sca }}\right) / n h$, where $n$ and $h$ are the number of particles per unit volume and thickness of the layer with the embedded scatters. For example, $C_{\text {sca }}$ for np-Si $+0.05 \mathrm{Au}\left(n=4 \times 10^{21} \mathrm{~m}^{-3}, h=550 \times\right.$ $10^{-9} \mathrm{~m}$ and $\left.I_{\text {sca }}=0.05\right)$ at $700 \mathrm{~nm}$ is about $1.4 \times 10^{-15} \mathrm{~m}^{2}$. The Comsol simulation suggests that embedded Au particles have the scattering cross-section of $3.2 \times 10^{-15} \mathrm{~m}^{2}$. The discrepancy might arise from the fact that the absorption of the np-Si matrix is not accounted in the simulation and only the backward scattering is collected by the integrating sphere. These two reasons cause the decrease of the experimental scattered intensity and lead to the underestimation of the cross-section.

The composite materials consisting of a porous silicon scaffold with embedded gold nanoparticles, benefitting from the possibility of controlling a few structural parameters, such as porosity, sizes of the pores and particles, thereby determining the Localised Surface Plasmon (LSP) properties. Namely, the porosity controls the local dielectric environment surrounding the particles and interparticle space. The density, size and shape of the particles can be regulated by the duration of the impregnation process, while their upper size is limited by the pore's diameter. Using a silicon scaffold with low pore size and density, the LSP resonance will be similar to that of an individual particle, but red-shifted with respect to vacuum or solvent with a low dielectric function. On the other hand, higher porosity and pore size offers an alternative to growing particles whose size can be controlled by the impregnation time. In such an environment, smaller particles behave as individual entities surrounded by the mix of silicon and air, while, for larger particles, their size 
Table 2 Simulation parameters

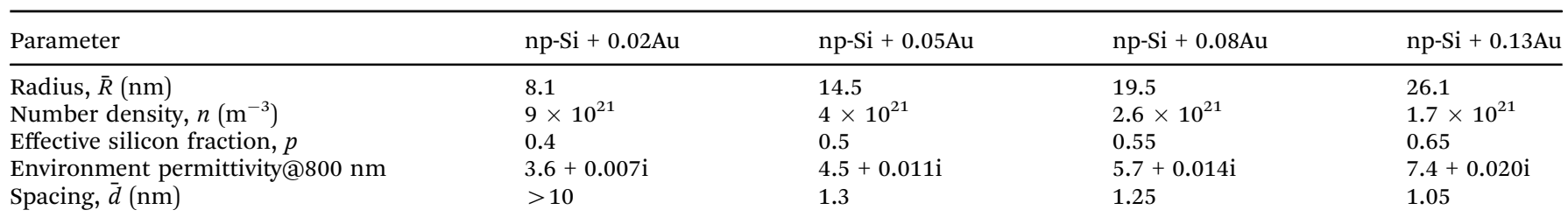

is set by the pore's diameter within the scaffold walls, tightly encompassing them with high dielectric index environment. The larger particles will not only have a red-shifted LSP spectrum, but their LSP fields can couple to produce hybridisation modes. Thus, when designing a nano-plasmonic composite material, one has some degrees of freedom to tune the appearance of the LSP spectra to a desired range of wavelengths. Fig. S7 in the ESI $\dagger$ demonstrates how the refractive index of $\mathrm{np}$-Si varies as a function of the porosity, as an example. By changing the anodization current and duration time, different porosity of np-Si samples can be obtained to tune the LSPR response.

\section{Use of Au/np-Si composites as a SERS substrate}

To demonstrate the advantage of the Au/np-Si composites, we employed the sample with the highest $\mathrm{Au}$ volume fraction, $f=0.13$, as the SERS substrate. A laser with $808 \mathrm{~nm}$ wavelength was applied to excite off-resonance Raman signals (see Methods for measurement details). Methylene Blue (MB) was used as a probe molecule, because its absorption peak is away from the excitation wavelength which minimises the parasitic luminescence background. ${ }^{50}$ To validate the effect, we compare in Fig. 3(a) the Raman spectra of the three samples consisting of bare np-Si, Au/np-Si composite without MB and Au/np-Si soaked in $1 \mu \mathrm{M}$ MB. All the samples show a strong and sharp Raman scattering at $520 \mathrm{~cm}^{-1}$ corresponding to the intrinsic TO phonon mode of silicon. ${ }^{51,52}$ Besides the phonon line, the background signal for the $\mathrm{Au} / \mathrm{np}-\mathrm{Si}$ is flat and featureless providing favourable conditions to use it as a SERS platform for chemical/biological detection. This is demonstrated by the Raman spectrum of the sample with the pores filled with $\mathrm{MB}$, which is recognised by the typical Raman peaks at 450,770 and $1150 \mathrm{~cm}^{-1}$ known from the literature. ${ }^{53-57}$ We note that to improve the presentation, the base lines of the spectra in Fig. 3(a) are arbitrarily located on the ordinate.

To establish the sensitivity of the method, we measured a series of samples with concentration range between $1 \mu \mathrm{M}$ and down to the ultra-low 1 pM, shown in Fig. 3(b), with the inset of the zoomed-in range between 400 and $500 \mathrm{~cm}^{-1}$. It can be seen that even for the concentration as low as $1 \mathrm{pM}$, the $\mathrm{MB}$ scattering peak at $450 \mathrm{~cm}^{-1}$ is still detectable, while the further increase of the concentration makes the MB spectral fingerprints more distinct. Fig. 3(c) shows the Raman intensity of the three representative peaks at 450,770 and $1150 \mathrm{~cm}^{-1}$ (located at short, middle and long spectrum region, respectively) as a function of $\mathrm{MB}$ concentration. It can be seen that the intensity increase for these peaks as a function of the concentration is identical. This suggests that the enhancement effect of a $\mathrm{Au} / \mathrm{np}$-Si composite does not have spectral selectivity. We note that multiple Raman measurements were taken over random locations on each sample to estimate the mean and deviation (Fig. S8 in the ESI $\dagger$ ). The curves shown in Fig. 2(b) are the averages of the Raman spectra measured in those locations. The error bars in Fig. 2(c) represent the peaks' deviation from their mean values.

The enhancement factor (EF) is an important criterion to evaluate quantitatively the performance of SERS substrates. ${ }^{34,35}$ As discussed in the literature, there are several ways to determine the EF. ${ }^{58-60}$ In this work, we adopted an approach that defines it as EF $=\frac{I_{\mathrm{SERS}} / N_{\mathrm{SERS}}}{I_{\mathrm{RS}} / N_{\mathrm{RS}}} \cdot{ }^{61} I_{\mathrm{SERS}}$ and $I_{\mathrm{RS}}$ are the Raman
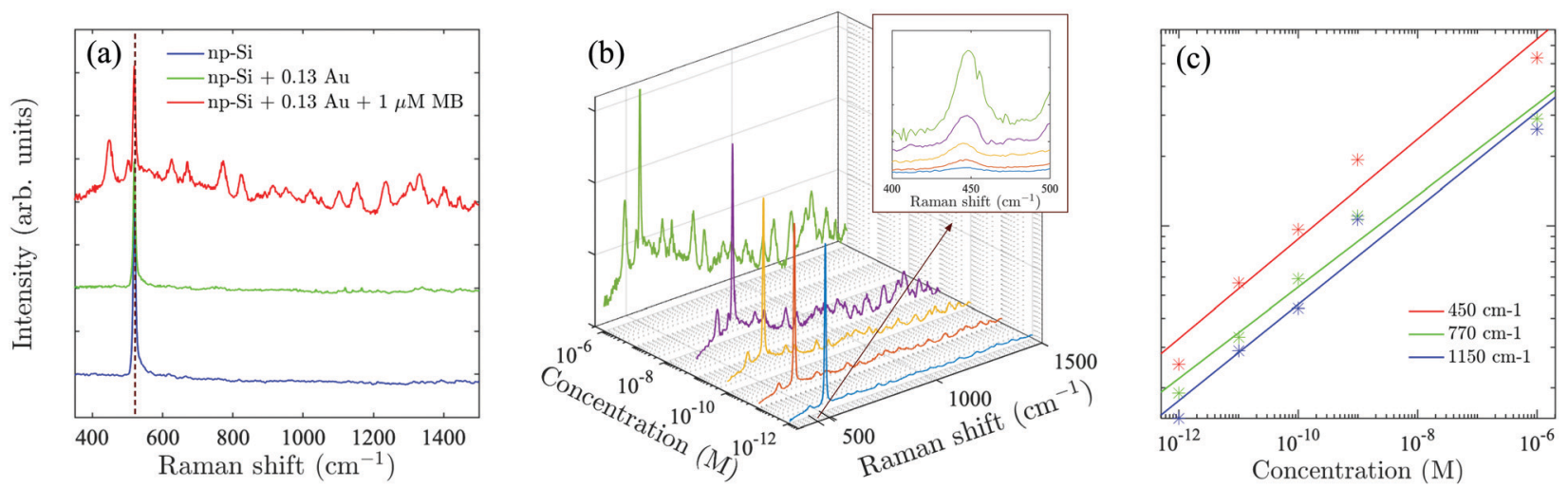

Fig. 3 (a) Raman spectra of $\mathrm{np}-\mathrm{Si}$ (blue), Au/np-Si (green) and Au/np-Si with $1 \mu \mathrm{M} \mathrm{MB}$ (red). The peaks marked by the dash line correspond to the TO phonon mode of silicon. The base lines of the spectra are arbitrarily located on the ordinate; (b) Raman spectra of Au/np-Si with different concentrations of $\mathrm{MB}$, ranging from $1 \mathrm{pM}$ to $1 \mu \mathrm{M}$. Inset: The enlarged view of the $450 \mathrm{~cm}^{-1}$ peak; (c) supralinear relationship of concentration and Raman intensity of three Raman peaks at 450 (red), 770 (green), 1150 (blue) $\mathrm{cm}^{-1}$, respectively. 
intensities with and without the surface enhancement; $N_{\text {SERS }}$ and $N_{\mathrm{RS}}$ are the corresponding number of analyte molecules exposed to the beam. For the reference measurement of $I_{\mathrm{RS}}$, a smooth Au film was used as a substrate which was uniformly covered by MB solution with concentration of $100 \mathrm{mM}$, corresponding to $2.4 \times 10^{12} \mathrm{MB}$ molecules (see Methods) in the area illuminated by the laser. As for the SERS measurement, there are about 24 molecules exposed to the laser at the lowest MB concentration ( $1 \mathrm{pM})$. The obtained SERS spectrum is shown by the blue line in Fig. 3(b). The reference Raman spectrum was recorded while keeping the power and spot size of the excitation laser the same as in SERS measurements, (Fig. S1 in the ESI $\dagger$ ). The intensity of $I_{\text {SERS }}$ and $I_{\text {RS }}$ was obtained by integrating the intensity of the three Raman peaks at 450, 770, and $1150 \mathrm{~cm}^{-1}$ which yields 0.196 and 4.063 , respectively (abs. unit), and the EF of $4.84 \times 10^{9}$. This is an ample EF value befitting the single molecule detection by the SERS method. ${ }^{34,62}$ The performance of our substrate approaches that of 3D plasmonic metamaterials with hierarchically ordered porous gold membranes consisting of close-packed arrays of nanoholes and uniformly distributed mesopores over the bulk, where a detection limit down to $10^{-13} \mathrm{M}$ was demonstrated for non-resonant Raman scattering from benzenethiol molecules. ${ }^{63}$

Typically, the EF for an isolated $\mathrm{Au}$ nanosphere is in the order of $10^{2}-10^{4},{ }^{64-66}$ which can be further improved to $10^{5}-10^{8}$ by changing its shape, ${ }^{67}$ the inter-particle spacing ${ }^{64,68,69}$ or distribution on a substrate. ${ }^{70}$ The high EF for our SERS substrate can be attributed to the peculiarity of a sponge-like morphological structure of np-Si. Unlike planar substrates used in previous studies, it exhibits an enormous surface for the adsorption of the analyte ${ }^{71}$ and accommodated gold nanoparticles. The analyte is not only detected on a facet surface of the substrate, but, to a greater extent, across the inner surface of np-Si pore walls. The large surface area of the pores traversing the volume can accommodate the outstanding density of the tightly packed clusters with a large cross-section. These conditions not only allow efficient LSP excitation across the layer depth, but create a large number of 'hot spots' where the fields further amplified in narrow gaps between interacting particles. Indeed, Table 2 proposes that particles with the radius as large as $26 \mathrm{~nm}$ can be fixed in space with in-between gaps between them of just a few nanometres. Moreover, it is possible to achieve conditions with the interacting particles dominating the assembly. The corresponding spectrum of Fig. 2(e and f) suggests that such tightly interacting particles dominate the response. On the other hand, the material can bind a high density of analyte molecules on the pores surface, in particular in the vicinity of the 'hot spots'. A similar arrangement is nearly impossible to realise in a liquid phase as the particles will coalesce in that state. Although it is possible to anchor the particles creating narrow gaps on the surface, the number of available sites is limited by the system's low dimensionality. Thus, the 3D np-Si scaffolding with a high density of interacting clusters yields a higher probability of finding molecules in gap areas than in its 2D counterpart and one would, therefore, expect higher overall contribution of amplified SERS response.

\section{Conclusions}

$\mathrm{Au} / \mathrm{np}$-Si composites with varying Au volume fraction were fabricated through the immersion plating technique. The AuNPs were successfully embedded into the 550 nm-thick np-Si substrates, forming a 3D metal/semiconductor composite material. SEM, TEM and EDX analysis confirmed the presence of AuNPs uniformly distributed over the volume. The analysis was used to obtain the average AuNP size and their density, which were employed to simulate LSPR scattering spectra. The numerical modelling suggests that the spectra are characterised by single particle and inter-particle coupling resonances. We examined the potential application to use the composites as a SERS substrate. We demonstrated that the concentration as low as a few picomolar can be detected for the benchmark compound of methylene blue. Our estimates suggest that the enhancement factor of $10^{9}$ can be achieved on these composite materials. The reason for such high amplification might be attributed to the peculiarities of np-Si. The material can accommodate a large number of metal particles interacting over the entire volume of the substrate containing analyte molecules absorbed in the pores.

\section{Methods}

\section{VIS-NIR spectrometer}

The back-scattered light was collected by a PerkinElmer Lambda 1050 UV/Vis/NIR spectrometer equipped with an integrating sphere module. During the measurement, the broad-band, unpolarised halogen light source was used to illuminate the samples. The samples were placed on a port where only the back-reflected light was collected inside the integrating sphere. To exclude the specular component from the diffuse scattered reflectance, a corresponding aperture was opened on the port which allowed the specularly reflected light to exit the integrating sphere.

\section{Surface enhanced Raman spectroscopy}

An 808 nm laser (Changchun New Industries Optoelectronics Tech. Co., Ltd) was used as the excitation source through a $40 \times$ objective (NA 0.6, Plan-Neofluar, Zeiss). The back-scattered Raman signal was collected via the objective after passing through the notch filter (TECHSPEC 808 notch filter, Edmund Optics Inc., Germany). The signal was detected by a spectrograph (Acton Spectra Pro 2500i, Princeton Instruments, Trenton, NJ, USA) equipped with an electron-multiplying charge-coupled device (EMCCD) camera (iXon, Andor, Belfast, $\mathrm{UK}) . \mathrm{Au} / \mathrm{np}$-Si samples with the highest $\mathrm{Au}$ volume fraction, $f=0.13$, were used as the SERS substrate, while the reference substrate was fabricated by evaporating $100 \mathrm{~nm}$ gold onto a $1 \mathrm{~cm}^{2}$ glass slice in a vacuum. Both the Au/np-Si and reference samples were immersed in $2 \mathrm{~mL}$ Methylene Blue (MB, high purity, Alfa Aesar) solution. The SERS samples were soaked in solutions with various concentrations in the range between $1 \mathrm{pM}$ and $1 \mu \mathrm{M}$, while for the reference the whole volume of $100 \mathrm{mM}$ solution was dropped on the surface. All the samples 
were dried on a hotplate at $60{ }^{\circ} \mathrm{C}$ for $10 \mathrm{~min}$ in air. Assuming that all the MB molecules are uniformly adsorbed by the sample, there are $\sim 2.4 \times 10^{12}$ and $\sim 24 \mathrm{MB}$ molecules on the reference surface and in $\mathrm{Au} / \mathrm{np}-\mathrm{Si}$ volume for $1 \mathrm{pM} \mathrm{MB}$ concentration, respectively, exposed to the area of $2 \mu \mathrm{m}^{2}$ illuminated by the laser. The obtained Raman spectra for the samples with $\mathrm{Au} / \mathrm{np}-\mathrm{Si}$ as the substrate are shown in Fig. 3(b), while Fig. S1 in the ESI $\dagger$ shows that of the reference.

\section{Nano-porous silicon}

Nano-porous Si (np-Si) samples were prepared using electrochemical anodisation of a boron doped $\mathrm{Si}(100)$ wafer with resistivity in the range of $5-20 \mathrm{~m} \Omega \mathrm{cm}$, corresponding to a dopant density of $3 \times 10^{18} \mathrm{~cm}^{-3}$. A $1: 1$ ratio mixture of methanol and $40 \%$ hydrofluoric acid was used as the electrolyte at a current density of $100 \mathrm{~mA} \mathrm{~cm} \mathrm{~cm}^{-2}$ for $8 \mathrm{~s}$ to yield an np-Si layer with thickness of approximately $550 \mathrm{~nm}$ (measured from the cross-section of the SEM image). The wafer was then rinsed in methanol and dried in air. A $65 \%$ porosity was estimated using the gravimetric method. ${ }^{72}$ The structure of the samples before impregnation can be described as being of a sponge silicon material, consisting of thin flat wires interwoven to form the walls of hollow channels, running along the depth coordinate. The mean thickness of the walls was $10 \mathrm{~nm}$ and the diameter of the channels was about $50 \mathrm{~nm}$. The substrates used to fabricate the samples in this study were different from those usually employed to investigate photoluminescence, for which p-silicon substrates are generally better suited. ${ }^{73}$ Instead, our main focus here was to obtain luminescence-free, optically uniform membranes for which $\mathrm{p}+$ substrates are an established choice. ${ }^{74}$ For this work, the thickness of the layer was kept sufficiently thin to assure uniform penetration of the gold solution into the depth, while, on the other hand, remaining thick enough to provide an adequate depth to accommodate the laser penetration depth for Raman measurements (see Fig. S9, ESI $\dagger$ ). We note that this process could be developed to impregnate layers and membranes with a thickness of more than a hundred microns. With such flexibility one can design an impregnated layered material facilitating an interferometric amplification, ${ }^{33}$ in addition to SERS.

\section{Gold cluster impregnation}

The electroless immersion plating technique was used to impregnate gold clusters into the np-Si layer. The immersion solution was prepared by dissolving $9.85 \mathrm{mg}$ of tetrachloroauric acid $\left(\mathrm{HAuCl}_{4}\right)$ in $50 \mathrm{~mL}$ of ethanol, resulting in a concentration of $0.5 \mathrm{mM}$. Before the impregnation process, the np-Si sample was dipped in $2 \%$ of HF (diluted in DI water) for 30 seconds to remove the native oxide layer developed on the surface of the sample and inside the pores. Afterwards, the np-Si sample was immersed in the pre-mixed $\mathrm{HAuCl}_{4}$ solution and placed in an ultrasonic bath to agitate the solution and stimulate it to flow into the pores. Due to the native surface of pSi which contains metastable $\mathrm{Si}-\mathrm{H}_{x}$ termination, the following reaction was triggered near the pore interface: $\left[\mathrm{AuCl}_{4}\right]^{-}+\mathrm{H}^{+}+3 \mathrm{e}^{-}=\mathrm{Au}+4 \mathrm{Cl}^{-}+\mathrm{H}^{+}$, causing the reduction of $\mathrm{Au}$ and formation of $\mathrm{Au}$ nanoparticles.
After a chosen time period, the sample was rinsed in ethanol and dried in air. Fig. S3 (ESI $\dagger$ ) shows the SEM cross section image of sample np-Si $+0.02 \mathrm{Au}$, and $\mathrm{Au}$ clusters can be seen inside the np-Si pore channels. The analysis of the SEM image suggests that the mean diameter of the clusters in this sample is about $20 \mathrm{~nm}$. The picked points in the figure are the random point analysis of a $\mathrm{Au}$ nanoparticle, where Da shows the diameter and $\mathrm{Db}$ is the area of the selected $\mathrm{Au}$ nanoparticle.

\section{FEM simulation of single and double AuNP}

Comsol Mulitiphysics was used for the simulation of the scattering cross section of single and double $\mathrm{Au}$ particles. The particle size was set to $8.1,14.5,19.5$, and $26.1 \mathrm{~nm}$, respectively, surrounded by a homogeneous material with refractive index of np-Si. The excitation field was set to have both TE and TM components, in order to simulate the experimental condition to a great extent. The wavelength was set to sweep from 500 to $1400 \mathrm{~nm}$. The simulation suggested that the Bruggeman model is only suitable for samples with small Au volume fraction. A correction on the environment dielectric function was applied to fit with the experimental results for high $\mathrm{Au}$ volume fraction samples. It also confirmed that the second scattering peak in the measured scattering spectrum came from the coupling between two Au clusters with short separation distance.

\section{Conflicts of interest}

There are no conflicts to declare.

\section{Acknowledgements}

The authors thank Dstl for financial support enabled by the grant DSTLX100099482. The authors also thank Alexander Pattison for conducting the TEM experiment.

\section{Notes and references}

1 V. G. Kravets, A. V. Kabashin, W. L. Barnes and A. N. Grigorenko, Chem. Rev., 2018, 118, 5912-5951.

2 K. E. Fong and L.-Y. L. Yung, Nanoscale, 2013, 5, 12043-12071.

3 H. Yu, Y. Peng, Y. Yang and Z.-Y. Li, npj Comput. Mater., 2019, 5, 1-14.

4 H. Chen, X. Kou, Z. Yang, W. Ni and J. Wang, Langmuir, 2008, 24, 5233-5237.

5 S. Zeng, D. Baillargeat, H.-P. Ho and K.-T. Yong, Chem. Soc. Rev., 2014, 43, 3426-3452.

6 J.-F. Masson, Analyst, 2020, 145, 3776-3800.

7 N. Zhou, V. López-Puente, Q. Wang, L. Polavarapu, I. Pastoriza-Santos and Q.-H. Xu, $R S C A d v$., 2015, 5, 29076-29097.

8 A. Aubry, D. Y. Lei, A. I. Fernández-Domnguez, Y. Sonnefraud, S. A. Maier and J. B. Pendry, Nano Lett., 2010, 10, 2574-2579.

9 Y. Y. Lee, R. M. Kim, S. W. Im, M. Balamurugan and K. T. Nam, Nanoscale, 2020, 12, 58-66. 
10 W. P. Hall, J. N. Anker, Y. Lin, J. Modica, M. Mrksich and R. P. Van Duyne, J. Am. Chem. Soc., 2008, 130, 5836-5837.

11 Y. Leroux, J. C. Lacroix, C. Fave, V. Stockhausen, N. Félidj, J. Grand, A. Hohenau and J. R. Krenn, Nano Lett., 2009, 9, 2144-2148.

12 J. Ki, H. Y. Lee, H. Y. Son, Y.-M. Huh and S. Haam, ACS Appl. Mater. Interfaces, 2019, 11, 18923-18929.

13 A. J. Haes, L. Chang, W. L. Klein and R. P. Van Duyne, J. Am. Chem. Soc., 2005, 127, 2264-2271.

14 D. Furtado, M. Björnmalm, S. Ayton, A. I. Bush, K. Kempe and F. Caruso, Adv. Mater., 2018, 30, 1801362.

15 M. J. Natan, M. D. Musick, C. Keating and K. R. Brown, SERS substrates formed by hydroxylamine seeding of colloidal metal nanoparticle monolayers, US Pat., 6624886, 2003.

16 T. R. Jensen, M. D. Malinsky, C. L. Haynes and R. P. Van Duyne, J. Phys. Chem. B, 2000, 104, 10549-10556.

17 F.-C. Chen, J.-L. Wu, C.-L. Lee, Y. Hong, C.-H. Kuo and M. H. Huang, Appl. Phys. Lett., 2009, 95, 182.

18 M. Kahraman, E. R. Mullen, A. Korkmaz and S. Wachsmann-Hogiu, Nanophotonics, 2017, 6, 831-852.

19 G. Demirel, H. Usta, M. Yilmaz, M. Celik, H. A. Alidagi and F. Buyukserin, J. Mater. Chem. C, 2018, 6, 5314-5335.

20 I. Pastoriza-Santos, C. Kinnear, J. Pérez-Juste, P. Mulvaney and L. M. Liz-Marzán, Nat. Rev. Mater., 2018, 3, 375-391.

21 Z. Y. Bao, D. Y. Lei, R. Jiang, X. Liu, J. Dai, J. Wang, H. L. W. Chan and Y. H. Tsang, Nanoscale, 2014, 6, 9063-9070.

22 J. Sasai and K. Hirao, J. Appl. Phys., 2001, 89, 4548-4553.

23 R. Cui, C. Liu, J. Shen, D. Gao, J.-J. Zhu and H.-Y. Chen, Adv. Funct. Mater., 2008, 18, 2197-2204.

24 N. Pinçon, B. Palpant, D. Prot, E. Charron and S. Debrus, Eur. Phys. J. D, 2002, 19, 395-402.

25 N. Waiskopf, Y. Ben-Shahar and U. Banin, Adv. Mater., 2018, 30, 1706697.

26 G. Yu, X. Wang, J. Cao, S. Wu, W. Yan and G. Liu, Chem. Commun., 2016, 52, 2394-2397.

27 K. H. W. Ho, A. Shang, F. Shi, T. W. Lo, P. H. Yeung, Y. S. Yu, X. Zhang, K.-y. Wong and D. Y. Lei, Adv. Funct. Mater., 2018, 28, 1800383.

28 T. Kormilina, E. Stepanidenko, S. Cherevkov, A. Dubavik, M. Baranov, A. Fedorov, A. Baranov, Y. Gun'Ko and E. Ushakova, J. Mater. Chem. C, 2018, 6, 5278-5285.

29 J. Li, S. K. Cushing, P. Zheng, T. Senty, F. Meng, A. D. Bristow, A. Manivannan and N. Wu, J. Am. Chem. Soc., 2014, 136, 8438-8449.

30 J.-H. Zhong, J. Vogelsang, J.-M. Yi, D. Wang, L. Wittenbecher, S. Mikaelsson, A. Korte, A. Chimeh, C. L. Arnold, P. Schaaf, E. Runge, A. L'Huillier, A. Mikkelsen and C. Lienau, Nat. Commun., 2020, 11, 1-10.

31 L. Canham, Handbook of porous silicon, Springer, 2014.

32 A. Zakar, R. Wu, D. Chekulaev, V. Zerova, W. He, L. Canham and A. Kaplan, Phys. Rev. B, 2018, 97, 155203.

33 W. He, R. Wu, I. V. Yurkevich, L. T. Canham and A. Kaplan, Sci. Rep., 2018, 8, 1-7.

34 E. Le, Ru, E. Blackie, M. Meyer and P. G. Etchegoin, J. Phys. Chem. C, 2007, 111, 13794-13803.
35 P. Mao, C. Liu, G. Favraud, Q. Chen, M. Han, A. Fratalocchi and S. Zhang, Nat. Commun., 2018, 9, 1-8.

36 N. R. Jana, L. Gearheart and C. J. Murphy, J. Phys. Chem. B, 2001, 105, 4065-4067.

37 M. Grzelczak, J. Pérez-Juste, P. Mulvaney and L. M. LizMarzán, Chem. Soc. Rev., 2008, 37, 1783-1791.

38 D. E. Newbury, Scanning: The Journal of Scanning Microscopies, 2009, 31, 91-101.

39 K. Minami, Y. Saito, H. Kai, K. Shirota and K. Yada, J. Phys.: Conf. Ser., 2009, 012010.

40 G.-C. Li, Y.-L. Zhang and D. Y. Lei, Nanoscale, 2016, 8, 7119-7126.

41 H. Frohlich, Theory of Dielectrics, 2nd edn, 1958, vol. 57, pp. 592-601.

42 S. Nath, S. K. Ghosh, S. Praharaj, S. Panigrahi, S. Basu and T. Pal, New J. Chem., 2005, 29, 1527-1534.

43 P. Nordlander, C. Oubre, E. Prodan, K. Li and M. Stockman, Nano Lett., 2004, 4, 899-903.

44 W. Rechberger, A. Hohenau, A. Leitner, J. Krenn, B. Lamprecht and F. Aussenegg, Opt. Commun., 2003, 220, 137-141.

45 R. Wu, T. Mathieu, C. J. Storey, Q. Jin, J. Collins, L. T. Canham and A. Kaplan, Adv. Opt. Mater., 2021, 9, 2002119.

46 P. B. Johnson and R.-W. Christy, Phys. Rev. B, 1972, 6, 4370.

47 D. Bruggeman, Ann. Phys. Leipzig, 1935, 24, 636.

48 M. Khardani, M. Bouacha and B. Bessas, Phys. Status Solidi C, 2007, 4, 1986-1990.

49 C. F. Bohren and D. R. Huffman, Absorption and Scattering of light by small particles, John Wiley and Sons, Inc., NY, 1983.

50 G.-N. Xiao and S.-Q. Man, Chem. Phys. Lett., 2007, 447, 305-309.

51 A. Kaminska, O. Inya-Agha, R. J. Forster and T. E. Keyes, Phys. Chem. Chem. Phys., 2008, 10, 4172-4180.

52 P. Spizzirri, J.-H. Fang, S. Rubanov, E. Gauja and S. Prawer, 2010, arXiv preprint arXiv:1002.2692.

53 C. Li, Y. Huang, K. Lai, B. A. Rasco and Y. Fan, Food Control, 2016, 65, 99-105.

54 C. Srichan, M. Ekpanyapong, M. Horprathum, P. Eiamchai, N. Nuntawong, D. Phokharatkul, P. Danvirutai, E. Bohez, A. Wisitsoraat and A. Tuantranont, Sci. Rep., 2016, 6, 23733.

55 S. D. Roy, P. Sett, M. Ghosh and J. Chowdhury, J. Raman Spectrosc., 2017, 48, 38-45.

56 R. R. Naujok, R. V. Duevel and R. M. Corn, Langmuir, 1993, 9, 1771-1774.

57 K.-D. Shim and E.-S. Jang, Bull. Korean Chem. Soc., 2018, 39, 936-940.

58 Y. Wang, H. Chen, S. Dong and E. Wang, J. Chem. Phys., 2006, 124, 074709.

59 M. Green and F. M. Liu, J. Phys. Chem. B, 2003, 107, 13015-13021.

60 K. Wu, L. Q. Nguyen, T. Rindzevicius, S. S. Keller and A. Boisen, Adv. Opt. Mater., 2019, 7, 1901413.

61 E. C. Le Ru and P. G. Etchegoin, MRS Bull., 2013, 38, 631.

62 E. J. Blackie, E. C. Le Ru and P. G. Etchegoin, J. Am. Chem. Soc., 2009, 131, 14466-14472.

63 X. Zhang, Y. Zheng, X. Liu, W. Lu, J. Dai, D. Y. Lei and D. R. MacFarlane, Adv. Mater., 2015, 27, 1090-1096. 
64 L. Litti and M. Meneghetti, Phys. Chem. Chem. Phys., 2019, 21, 15515-15522.

65 D.-S. Wang and M. Kerker, Phys. Rev. B, 1981, 24, 1777.

66 V. Joseph, A. Matschulat, J. Polte, S. Rolf, F. Emmerling and J. Kneipp, J. Raman Spectrosc., 2011, 42, 1736-1742.

67 C. Hrelescu, T. K. Sau, A. L. Rogach, F. Jäckel and J. Feldmann, Appl. Phys. Lett., 2009, 94, 153113.

68 S. L. Kleinman, B. Sharma, M. G. Blaber, A.-I. Henry, N. Valley, R. G. Freeman, M. J. Natan, G. C. Schatz and R. P. Van Duyne, J. Am. Chem. Soc., 2013, 135, 301-308.

69 S. Hong and X. Li, J. Nanomater., 2013, 2013, 790323, DOI: $10.1155 / 2013 / 790323$.
70 A. Wei, B. Kim, B. Sadtler and S. L. Tripp, Chem. Phys. Chem., 2001, 2, 743-745.

71 S. A. Trauger, E. P. Go, Z. Shen, J. V. Apon, B. J. Compton, E. S. Bouvier, M. Finn and G. Siuzdak, Anal. Chem., 2004, 76, 4484-4489.

72 R. Wu, J. Collins, L. Canham and A. Kaplan, Appl. Sci., 2018, 8, 1810.

73 J. Joo, T. Defforge, A. Loni, D. Kim, Z. Li, M. J. Sailor, G. Gautier and L. T. Canham, Appl. Phys. Lett., 2016, 108, 153111.

74 A. Campos, J. Torres and J. Giraldo, Surf. Rev. Lett., 2002, 9, 1631-1635. 